BIOLOGICAL CRYSTALLOGRAPHY

ISSN 1399-0047

Received 28 August 2014

Accepted 17 January 2015

Edited by S. Wakatsuki, Stanford

University, USA

Keywords: radiation damage; dose limit; energy dependence.

\section{Radiation decay of thaumatin crystals at three X-ray energies}

\author{
Dorothee Liebschner, ${ }^{a}$ Gerold Rosenbaum, ${ }^{\mathrm{b}}$ Miroslawa Dauter ${ }^{\mathrm{c}}$ and Zbigniew \\ Dauter $^{\text {d* }}$
}

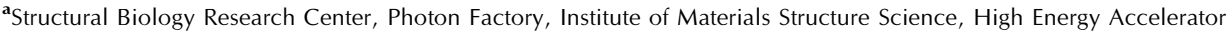
Research Organization, Tsukuba, Japan, ${ }^{\mathbf{b}}$ Department of Biochemistry, University of Georgia and Structural Biology Center, Argonne National Laboratory, Argonne, IL 60439, USA, 'Leidos Biomedical Research Inc., Basic Science Program, Argonne National Laboratory, Argonne, IL 60439, USA, and 'd Synchrotron Radiation Research Section, MCL, National Cancer Institute, Argonne National Laboratory, Argonne, IL 60439, USA. *Correspondence e-mail: dauter@anl.gov
\end{abstract}

Radiation damage is an unavoidable obstacle in X-ray crystallographic data collection for macromolecular structure determination, so it is important to know how much radiation a sample can endure before being degraded beyond an acceptable limit. In the literature, the threshold at which the average intensity of all recorded reflections decreases to a certain fraction of the initial value is called the 'dose limit'. The first estimated $D_{50}$ dose-limit value, at which the average diffracted intensity was reduced to $50 \%$, was $20 \mathrm{MGy}$ and was derived from observing sample decay in electron-diffraction experiments. A later X-ray study carried out at $100 \mathrm{~K}$ on ferritin protein crystals arrived at a $D_{50}$ of $43 \mathrm{MGy}$, and recommended an intensity reduction of protein reflections to $70 \%, D_{70}$, corresponding to an absorbed dose of $30 \mathrm{MGy}$, as a more appropriate limit for macromolecular crystallography. In the macromolecular crystallography community, the rate of intensity decay with dose was then assumed to be similar for all protein crystals. A series of diffraction images of cryocooled $(100 \mathrm{~K})$ thaumatin crystals at identical small, $2^{\circ}$ rotation intervals were recorded at X-ray energies of $6.33,12.66$ and $19.00 \mathrm{keV}$. Five crystals were used for each wavelength. The decay in the average diffraction intensity to $70 \%$ of the initial value, for data extending to $2.45 \AA$ resolution, was determined to be about 7.5 MGy at $6.33 \mathrm{keV}$ and about $11 \mathrm{MGy}$ at the two higher energies.

\section{Introduction}

$\mathrm{X}$-ray photons, which give rise to the diffraction patterns used for structure determination, are also absorbed by the crystalline sample, thus creating a cascade of damaging processes. As a consequence, the structure of the sample changes, accompanied by a change in the diffraction intensities, as reported early on by Blake \& Phillips (1962). In contrast to the individual diffraction intensities, which may increase or decrease, the average intensity monotonically decreases as the $\mathrm{X}$-ray dose increases. At a certain level of decay, structure solution and biological interpretation become unreliable (Blake \& Phillips, 1962; Hendrickson, 1976; Garman \& Nave, 2002; Ravelli \& Garman, 2006; Holton, 2009; Garman, 2010).

Besides the global decay of average diffraction intensities, specific structural changes occur, such as the breakage of disulfide bonds and the decarboxylation of glutamates and aspartates (Burmeister, 2000; Weik et al., 2000; Ravelli \& McSweeney, 2000). Although we do not analyze site-specific decay in this report, it should be noted that these local structural changes have been observed at much lower doses than those that induce noticeable global decay (Ravelli \& McSweeney, 2000; Weik et al., 2000). The review by Garman 
Table 1

Experimental conditions at the three different photon energies.

\begin{tabular}{|c|c|c|c|c|c|}
\hline Crystal & Rotation $\left({ }^{\circ}\right)$ & $\begin{array}{l}\text { Exposure } \\
\text { time } \dagger(\mathrm{s})\end{array}$ & $\begin{array}{l}\text { Detector } \\
\text { distance }(\mathrm{mm})\end{array}$ & $\begin{array}{l}\text { No. of } \\
\text { images }\end{array}$ & $\begin{array}{l}\text { Maximum } \\
\text { resolution }(\AA)\end{array}$ \\
\hline \multicolumn{6}{|c|}{$6.33 \mathrm{keV}(1.959 \AA)$} \\
\hline $\begin{array}{l}\text { xtal01 } \\
\text { xtal02 } \\
\text { xtal03 } \\
\text { xtal04 } \\
\text { xtal05 }\end{array}$ & 2 & 2 & 108 & 250 & 2.08 \\
\hline \multicolumn{6}{|c|}{$12.66 \mathrm{keV}(0.979 \AA)$} \\
\hline $\begin{array}{l}\text { xtal01 } \\
\text { xtal02 } \\
\text { xtal03 } \$ \\
\text { xtal04 } \\
\text { xtal05 }\end{array}$ & 2 & 2 & 150 & 1000 & 1.22 \\
\hline \multicolumn{6}{|c|}{$19.00 \mathrm{keV}(0.653 \AA)$} \\
\hline xtal01 & 2 & $5(20)$ & 230 & 200 & 1.22 \\
\hline xtal03 & & $5(20)$ & & & \\
\hline xtal04 & & $4(16)$ & & & \\
\hline xtal05 & & $4(16)$ & & & \\
\hline
\end{tabular}

$\dagger$ For $19 \mathrm{keV}$, the times in parentheses denote the exposure times for the unrecorded 'burn'. $\quad$ * xtal03 at $12.66 \mathrm{keV}$ was not used in the analysis since it moved partially out of the beam during the experiment.

(2010) provides an informative introduction to radiation damage in crystals of biological macromolecules. Apart from the global decay of intensities, other effects of radiation damage that can be readily derived from diffraction images are changes in unit-cell parameters, mosaicity and scaling $B$ factors. However, apart from the $B$ factors (Kmetko et al., 2006), they are not useful as metrics for radiation damage.

Cooling crystals to cryogenic temperatures extends their lifetime by a factor of 20-50 (Hope, 1988; Rodgers, 1994; Kmetko et al., 2006; Warkentin \& Thorne, 2010; Warkentin et al., 2013). However, the very high fluxes available at thirdgeneration storage rings make it possible to record diffraction data from more weakly diffracting samples, or at a higher resolution, in a reasonably short time, resulting in high radiation doses. For these reasons, radiation-damage research has again become a major concern in the biocrystallographic community. The first quantitative estimation of radiation damage was provided via electron-diffraction experiments performed by Henderson (1990), who estimated that the dose of energy absorbed by crystalline samples that diminished the average intensity of diffracted reflections to $50 \%, D_{50}$, equaled 20 MGy. He proposed this value as a dose limit for X-ray crystallography on cryocooled protein crystals. The results obtained by Owen et al. (2006) on the basis of X-ray diffraction data collected at $100 \mathrm{~K}$ from ferritin crystals suggested a $D_{50}$ value of $43 \mathrm{MGy}$. These authors suggested that a more appropriate criterion for the radiation-damage limit is $D_{70}$, the dose value that lowers the average intensity to $\ln (2) \simeq 0.70$. The value $D_{70}=30 \mathrm{MGy}$ is usually referred to as the 'Garman limit'.

Since June 1999, regular biannual meetings have summarized current research on X-ray radiation damage to biological samples (International Workshop on X-ray Radiation Damage to Biological Crystalline Samples, 1999-2014) and their proceedings have been published in special issues of the
Journal of Synchrotron Radiation (volume 9, part 6; volume 12, part 3; volume 14, part 1; volume 16, part 2; volume 18, part 3 ; volume 20, part 1).

While investigating the relation between diffraction data quality and photon energy, we noticed that the reflection intensities measured from crystals of thaumatin decayed with dose significantly more rapidly than expected. Thus, we decided to study these observations in more detail, since they suggested that there may be no generally applicable radiationdose limit and that different crystals may have different susceptibilities to radiation damage.

We recorded a series of diffraction images at identical $2^{\circ}$ rotation intervals at three different energies (6.33, 12.66 and $19.00 \mathrm{keV}$ ) and analyzed the data in terms of radiation damage. For each photon energy, we collected data from five different samples using the same protocol in order to ensure a certain degree of statistical validity of the results and to average the variations between individual samples.

\section{Materials and methods}

\subsection{Crystallization and data collection}

Thaumatin was chosen as a test protein because it is easily available and crystallizes readily. Crystals were grown by the hanging-drop method using a protein solution at approximately $35 \mathrm{mg} \mathrm{ml}^{-1}$ in $50 \mathrm{~m} M$ HEPES buffer $\mathrm{pH} 7.0$ mixed in a 1:1 ratio with well solution consisting of $0.75 \mathrm{M}$ sodium/ potassium tartrate, $0.1 M$ citrate buffer $\mathrm{pH}$ 6.5. For data collection, the crystals were cooled in a stream of gaseous nitrogen at $100 \mathrm{~K}$ delivered by an Oxford Cryosystems cryocooler. The cryosolution consisted of reservoir solution supplemented with $28 \%(v / v)$ glycerol and all crystals were briefly immersed (for 3-5 s) in it before being rapidly transferred to a goniostat. All crystals were tetragonal, belonging to space group $P 4_{1} 2_{1} 2$, with unit-cell parameters of about $a=b=58, c=150 \AA$.

Diffraction data were collected on beamline 19-ID of the Structural Biology Center at the Advanced Photon Source (APS), Argonne National Laboratory (Rosenbaum et al., 2006) using an ADSC Q315r CCD detector at X-ray energies of 19.00, 12.66 and $6.33 \mathrm{keV}$. The long $c$ cell dimension of each crystal was oriented approximately parallel to the detector plane in order to avoid overlap of reflection profiles on the detector. Diffraction images with the same starting crystal orientation and a rotation width of $2^{\circ}$ were recorded consecutively. This method of recording a series of diffraction images at identical small rotation intervals guarantees that the same crystal volume is always irradiated and minimizes the influence of the properties of the crystal and detector on the relative accuracy of recorded diffraction intensities of consecutive images (Liebschner et al., 2012; Sliz et al., 2003). The exposure time per frame was selected to keep the number of overloaded detector pixels at less than 40 in the first image. The total number of images was adjusted to the lifetime of the crystal, i.e. consecutive frames were recorded until the highresolution spots disappeared. At $19 \mathrm{keV}$, this procedure would 
Table 2

Parameters relevant for calculation of the dose with RADDOSE v.2 (Paithankar et al., 2009).

\begin{tabular}{|c|c|c|c|c|}
\hline Crystal & $\begin{array}{l}\text { Flux for first image } \\
\left(10^{10} \text { photons s }^{-1}\right)\end{array}$ & $\begin{array}{l}\text { Beam size } \dagger \\
(\mu \mathrm{m})\end{array}$ & $\begin{array}{l}\text { Crystal } \\
\text { dimensions } \ddagger \\
(\mu \mathrm{m})\end{array}$ & $\begin{array}{l}\text { Dose for } \\
\text { first image } \\
\text { (MGy) }\end{array}$ \\
\hline \multicolumn{5}{|c|}{$6.33 \mathrm{keV}(1.959 \AA)$} \\
\hline xtal01 & 12.0 & \multirow[t]{5}{*}{75,92} & $111,113,87$ & 0.068 \\
\hline xtal02 & 11.7 & & $105,152,99$ & 0.065 \\
\hline xtal03 & 12.3 & & $131,133,100$ & 0.069 \\
\hline xtal04 & 12.2 & & $110,107,126$ & 0.066 \\
\hline xtal05 & 11.7 & & $98,112,119$ & 0.064 \\
\hline \multicolumn{5}{|c|}{$12.66 \mathrm{keV}(0.979 \AA)$} \\
\hline xtal01 & 4.5 & \multirow[t]{4}{*}{51,29} & $99,145,120$ & 0.031 \\
\hline xtal02 & 5.4 & & $101,86,93$ & 0.038 \\
\hline xtal04 & 5.4 & & $111,120,116$ & 0.038 \\
\hline xtal05 & 5.2 & & $103,107,96$ & 0.037 \\
\hline \multicolumn{5}{|c|}{$19.0 \mathrm{keV}(0.653 \AA)$} \\
\hline xtal01 & 5.7 & \multirow[t]{5}{*}{44,39} & $147,125,112$ & 0.036 \\
\hline xtal02 & 5.8 & & $136,100,80$ & 0.036 \\
\hline xtal03 & 5.8 & & $111,107,129$ & 0.037 \\
\hline xtal04 & 5.8 & & $138,103,113$ & 0.029 \\
\hline xtal05 & 5.7 & & $144,125,113$ & 0.029 \\
\hline
\end{tabular}

$\dagger$ The beam size is given as the horizontal dimension followed by the vertical dimension. $\ddagger$ The crystal dimensions are given as horizontal, vertical and thickness values.

have led to a very large number of exposures before the crystals were exhausted. Therefore, a protocol consisting of a recorded exposure of 4 or $5 \mathrm{~s}$ followed by a four times longer unrecorded 'burn' exposure with the same X-ray flux was used. At $6.33 \mathrm{keV}$, the X-ray beam was attenuated by a $0.08 \mathrm{~mm}$ thick aluminium sheet (13\% transmission) in order to use an exposure time similar to that used for the other energies without creating too many overloaded pixels. Table 1 summarizes the experimental conditions for each crystal at each wavelength.

\subsection{Dose calculation}

The dose was estimated using RADDOSE v.2 (Paithankar $e t$ al., 2009). Table 2 summarizes the relevant dose-calculation parameters of the experiment, such as crystal dimensions, beam size and flux.

We used the horizontal and vertical focusing elements to form the beam profile into as rectangular ('top hat') a shape as possible in order to achieve a dose across the irradiated crystal volume that was as uniform as possible. Fig. 1 shows the typical horizontal and vertical beam profiles used, recorded by scanning a $13 \mu \mathrm{m}$ wide slit across the beam. The X-ray flux was measured using a dry, nitrogen-filled ionization chamber with an active length of $100 \mathrm{~mm}$. The recorded current was converted to the rate of incident photons using tabulated mass absorption coefficients of nitrogen (McMaster et al., 1969), assuming standard density and a work function of $34.6 \mathrm{eV}$ per electron-ion pair. For validation, a $12.6 \mathrm{keV}$ X-ray flux measured with the ion chamber was compared with the same flux measured with a calibrated Si PIN diode [DP00325, calibrated, with an uncertainty of $1 \%$, by the PhysikalischTechnische Bundesanstalt (PTB), Germany; on loan from the APS Detector Pool] and found to be in agreement within 5\%. The flux at the sample was derived from the flux measured with the ion chamber by correcting for all absorption between the location of the sample and the location of the collector plates in the ion chamber.

At low photon energies, absorption becomes noticeable and the flux along the beam path through the crystal can no longer be assumed to be constant. At $6.33 \mathrm{keV}$, the absorption of
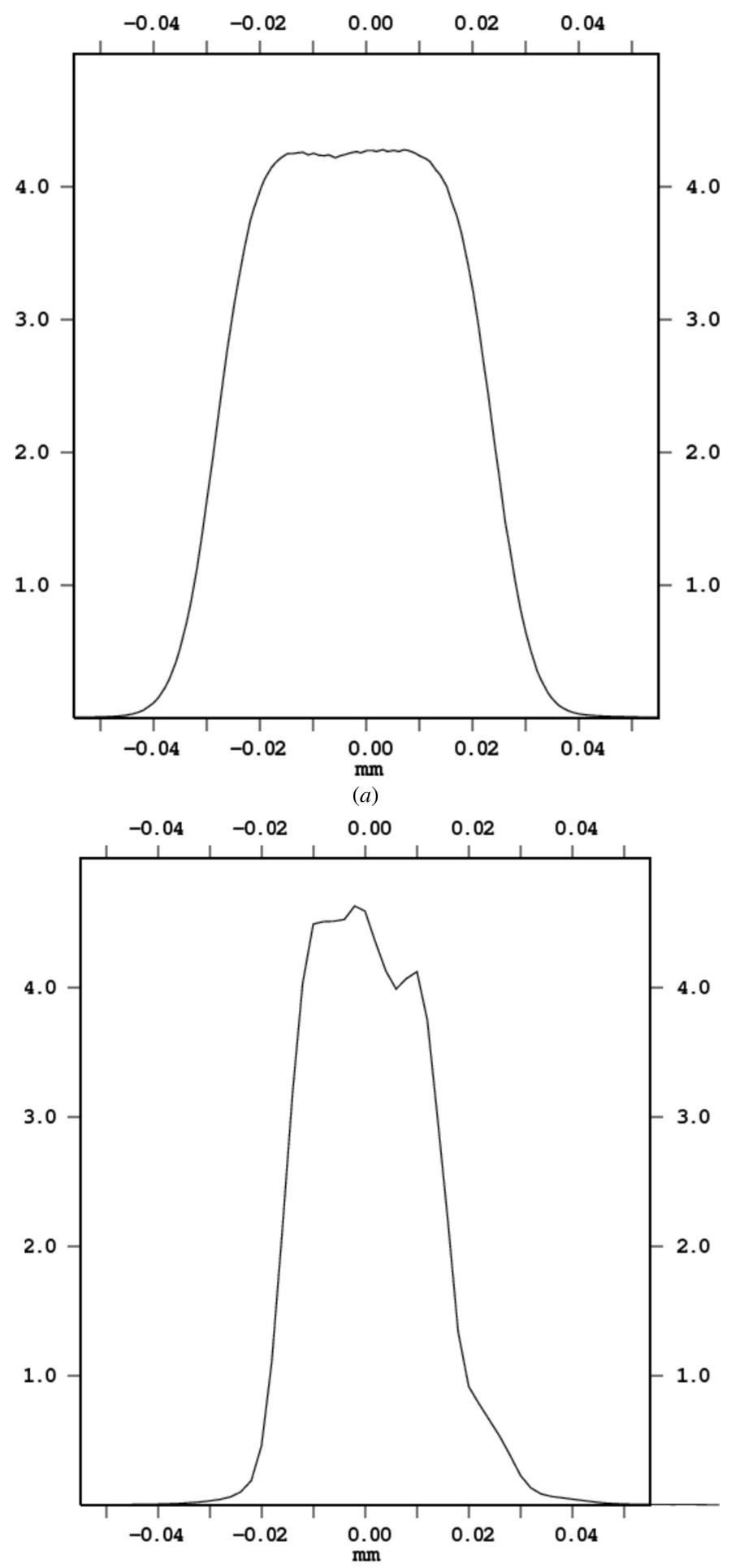

Figure 1

(b)

Horizontal ( $a$ ) and vertical (b) profiles of the beam used for the experiment at $12.66 \mathrm{keV}$. The vertical scale of the intensities is arbitrary. The beam profiles at 6.33 and $19.0 \mathrm{keV}$ were very similar. 
water (a reasonable surrogate for a protein crystal) is about $19 \%$ per $0.1 \mathrm{~mm}$ thickness. The resulting dose gradient is taken into account by RADDOSE as it calculates an average dose. It should be noted that the dose gradient adds to the uncertainty in the dose calculation as the three-dimensional shape of the crystal is only approximately known and is not an input parameter to RADDOSE. Not taken into account is the solvent layer in front of the crystal. Using pictures of the mounted samples, we determined the approximate thickness of the solvent layer, calculated the absorption coefficient of the solvent (using the web server at http://henke.lbl.gov/ optical_constants/) and corrected the flux accordingly. The possible inaccuracy of at most $20 \mu \mathrm{m}$ in the visual estimation of the solvent and crystal thickness may result in an uncertainty in the dose of $5 \%$ at $6.33 \mathrm{keV}$. At 12.66 and $19 \mathrm{keV}$, absorption is so small that these corrections would not be significant. The overall uncertainties in the dose estimations are therefore about $5 \%$ for 12.66 and $19 \mathrm{keV}$ and not more than $10 \%$ for $6.33 \mathrm{keV}$.

The small changes in flux on the sample that occurred during the course of the exposure series were recorded by a beam-position/beam-intensity monitor (BPM; Alkire et al., 2000) located downstream of the collimating slits. The beamintensity signal from the BPM that had been referenced against the ion-chamber current for each photon energy was used to adjust the dose value assigned to each image relative to the dose calculated for the first image.

\subsection{Data analysis}

The diffracted intensities were integrated with $D E N Z O$ (Otwinowski \& Minor, 1997) using the 'oscillation start X' command, which prevents the program from advancing the crystal rotation matrix between consecutive exposures. Furthermore, the mosaicity was deliberately overestimated and fixed to $0.5^{\circ}$ for each image in order to safely classify reflections marked as fully recorded ('fullys'). In addition,

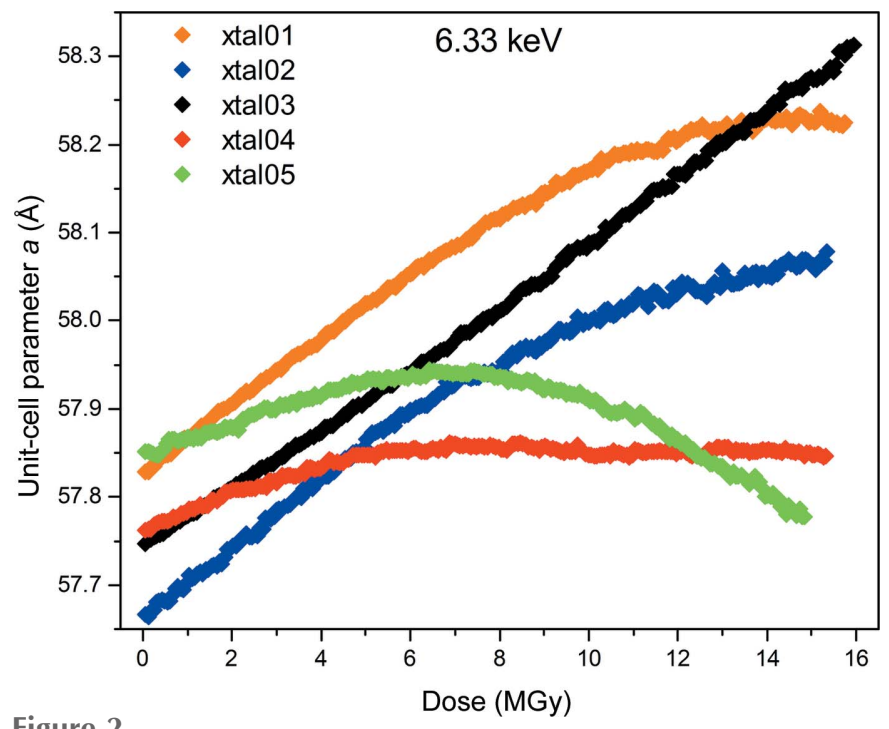

Figure 2

Change of the unit-cell parameter $a$ as a function of dose for the five crystals measured at $6.33 \mathrm{keV}$. The color code is indicated at the top left. since the unit-cell parameters of the crystals changed slightly with increasing dose (Fig. 2), we made sure that for each crystal only reflections that were fully recorded and present in all images were included in calculations. The intensities obtained from profile fitting and stored in the individual output $*$. $x$ files of each image were used for further analysis. The Lorentz and polarization corrections were not applied in the estimation of reflection intensities, since this analysis focused on the decay of diffracted intensities not structurefactor amplitudes. Average intensities were computed for the whole resolution range and in smaller resolution shells; the same limits were applied for all crystals at each energy.

\section{Results and discussion}

\subsection{Overall properties of the data sets}

The experiment yielded 15 sets of $2^{\circ}$ rotation images, with each set containing essentially the same reflections in each diffraction frame. As a result of the slowly changing mosaicity and unit-cell parameters, the images differed slightly in the occurrence of the partially recorded reflections. In the analysis, however, only reflections classified as safe 'fullys' were used. The only differences between the intensities of fully recorded reflections in consecutive images stem from decay owing to radiation damage. Integration of the data with $D E N Z O$ was successful and all fully recorded intensities from the output $*$.x files were analyzed in terms of radiation damage. However, after preliminary examination of the intensities, it was determined that xtal03 at $12.66 \mathrm{keV}$ probably slipped during data collection, so the data from this crystal were not used in this study.

The resolution of the data recorded at 12.66 and $19.0 \mathrm{keV}$ extended to $1.22 \AA$; however, at $6.33 \mathrm{keV}$ the minimum detector distance of $108 \mathrm{~mm}$ limited the resolution to $2.08 \AA$, even though the crystals were of good quality and would certainly have diffracted to higher resolution.

Table 2 lists the parameters relevant to the dose calculation (flux, beam size and crystal size) and the dose that the crystals received during the measurement of the first image of each series. The doses of the following images were prorated according to the small changes in flux recorded by the method described in $\S 2.2$.

\subsection{Decay of average intensities}

Fig. 3 shows the decay of the average intensity as a function of dose for the data sets recorded at $19.0 \mathrm{keV}$, calculated for the whole resolution range, which was $30-1.22 \AA$ at this energy. The curves are very smooth and do not display any discontinuities. The five crystals have different average intensities in the beginning, ranging from 2250 integrated units (IUs; i.e. the units of DENZO *.x files) for xtal04 to over 4000 IUs for xtal01. They decay to less than 700 IUs (xtal04) and 900 IUs (xtal01) after recording 200 images, which is far beyond the extent of decay investigated in this study. All five curves have the exponential shape observed in the first radiation-damage study by Blake \& Phillips (1962). 
Table 3

Doses at which the average intensities in the indicated resolution ranges have decayed to $70 \%\left(D_{70}\right)$ and to $50 \%\left(D_{50}\right)$ of the initial value.

The last column contains the relative diffractive efficiency of each crystal, derived from the average intensity of reflections recorded in the first image and normalized by flux and irradiated crystal volume. The uncertainty in the dose estimations is about $10 \%$ for the $6.33 \mathrm{keV}$ data and about $5 \%$ for the other energies.

\begin{tabular}{|c|c|c|c|c|c|c|c|c|}
\hline \multirow[b]{2}{*}{$\begin{array}{l}\text { Energy } \\
(\mathrm{keV})\end{array}$} & \multirow[b]{2}{*}{ Crystal } & \multicolumn{3}{|l|}{$D_{70}(\mathrm{MGy})$} & \multicolumn{3}{|c|}{$D_{50}$ (MGy) } & \multirow[b]{2}{*}{$\begin{array}{l}\text { Diffraction } \\
\text { efficiency }\end{array}$} \\
\hline & & $30-2.45 \AA$ & $30-2.08 \AA$ & $30-1.22 \AA$ & $30-2.45 \AA$ & $30-2.08 \AA$ & $30-1.22 \AA$ & \\
\hline \multirow[t]{6}{*}{6.33} & xtal01 & 6.7 & 6.5 & & 11.6 & 11.2 & & 2.5 \\
\hline & xtal02 & 6.7 & 6.5 & & 11.6 & 11.2 & & 1.3 \\
\hline & xtal03 & 8.4 & 8.0 & & 14.4 & 13.7 & & 1.8 \\
\hline & xtal04 & 8.2 & 7.8 & & 14.7 & 14.1 & & 1.7 \\
\hline & xtal05 & 7.5 & 7.2 & & 12.0 & 11.6 & & 1.6 \\
\hline & Average & 7.5 & 7.2 & & 12.8 & 12.4 & & \\
\hline \multirow[t]{5}{*}{12.66} & xtal01 & 12.0 & 10.7 & 9.3 & 22.3 & 20.6 & 17.9 & 2.9 \\
\hline & xtal02 & 11.5 & 10.5 & 9.0 & 21.6 & 20.0 & 17.6 & 3.4 \\
\hline & xtal04 & 11.5 & 10.4 & 9.2 & 20.1 & 18.4 & 16.7 & 2.4 \\
\hline & xtal05 & 9.4 & 8.5 & 7.4 & 17.5 & 16.0 & 14.3 & 1.4 \\
\hline & Average & 11.1 & 10.1 & 8.8 & 20.4 & 18.7 & 16.6 & \\
\hline \multirow[t]{6}{*}{19.0} & xtal01 & 11.0 & 9.6 & 8.0 & 20.3 & 18.3 & 15.8 & 3.8 \\
\hline & xtal02 & 12.1 & 10.6 & 8.5 & 21.2 & 19.1 & 16.1 & 4.3 \\
\hline & xtal03 & 12.5 & 11.2 & 10.1 & 21.8 & 20.2 & 18.6 & 2.1 \\
\hline & xtal04 & 10.5 & 9.3 & 7.6 & 17.8 & 16.2 & 13.6 & 2.6 \\
\hline & xtal05 & 11.0 & 9.6 & 8.2 & 19.0 & 17.1 & 14.8 & 2.9 \\
\hline & Average & 11.4 & 10.0 & 8.5 & 20.0 & 18.2 & 15.8 & \\
\hline
\end{tabular}

summarizes the values for $D_{70}$, at which the intensity is reduced to $70 \%$ of $I_{1}$, and $D_{50}$, at which the intensity is halved, for data extending to different highresolution limits: $2.45 \AA$, comparable to that used by Owen et al. (2006); $2.08 \AA$, the highest resolution limit common to all three energies; and $1.22 \AA$, the highest achieved limit for 12.66 and $19.0 \mathrm{keV}$.

For resolutions up to $2.45 \AA$, the average $D_{70}$ values are $7.5,11.1$ and 11.4 MGy for $6.33,12.66$ and $19.0 \mathrm{keV}$, respectively. For the resolution range 30-2.08 $\AA$ the corresponding values are 7.2, 10.1 and 10.0 MGy. Whereas $\mathrm{X}$-radiation at the two higher energies appears to incur comparable damage to thaumatin crystals, the dose tolerance at the lower photon energy is $30 \%$ lower.

Different investigations using various methodologies have reported both energy dependence and independence of radiation damage. Fourme et al. (2012) stated that the data-collection

Fig. 4 shows the decay of the average intensity for xtal01 at $12.66 \mathrm{keV}$ in different resolution shells and for the whole resolution range (30.0-1.22 $)$. The average intensities decrease monotonically with increasing resolution. Likewise, the slope of the decay increases monotonically with increasing resolution.

By dividing the average intensity of an image by the intensity $I_{1}$ recorded in the first image of the exposure series, the dose $D_{\text {lim }}$ at which the intensity falls off to a percentage (lim) of its initial value can be readily derived. Table 3

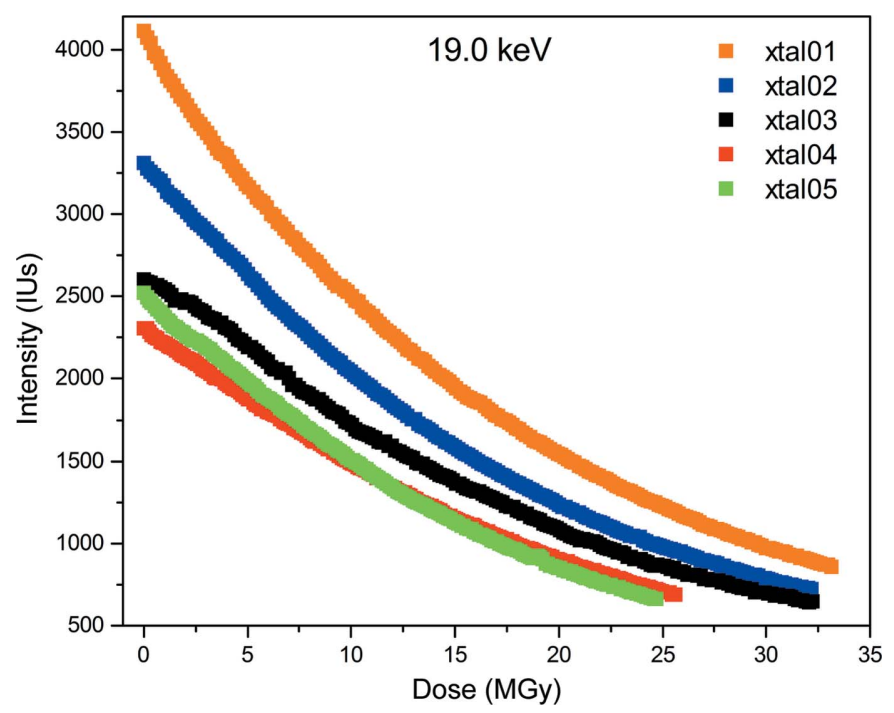

Figure 3

Average intensities as a function of dose for the data recorded at $19.0 \mathrm{keV}$. A resolution range of $30.0-1.22 \AA$ 为 was used for the calculation. The color code is indicated at the top right. efficiency increases about eight times when the photon energy changes from 8 to $30 \mathrm{keV}$. In contrast, Homer et al. (2011) found that site-specific damage at disulfide-bridge sites is greater at $14 \mathrm{keV}$ than at $9 \mathrm{keV}$, although methionine $\mathrm{S}$ atoms display no energy dependence. Shimizu et al. (2007) studied radiation damage of HEWL crystals over an energy range from 6.5 to $33 \mathrm{keV}$. Using criteria such as changes in mosaicity,

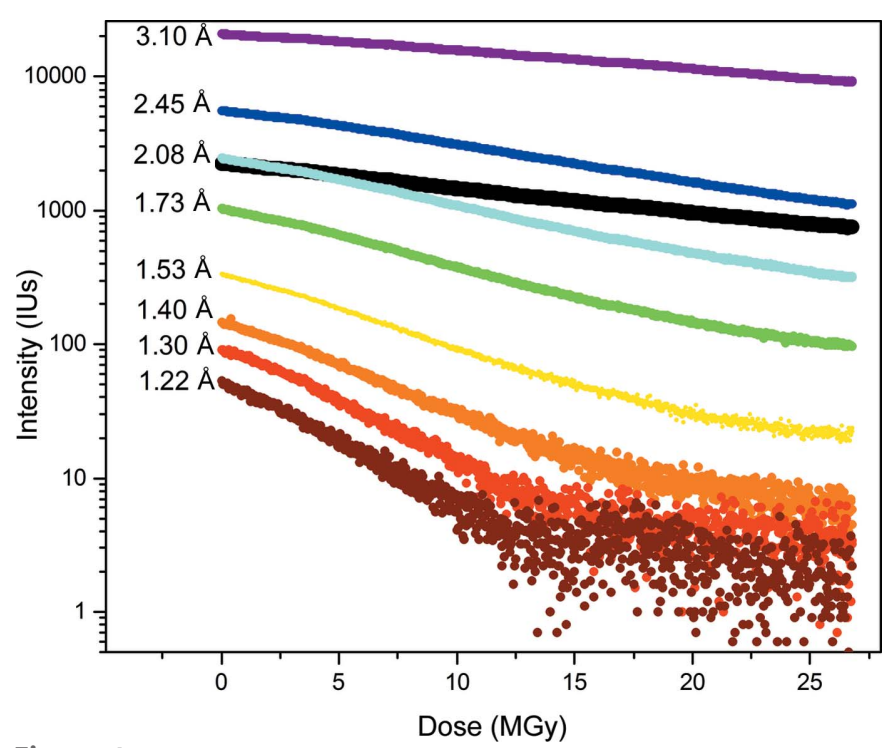

Figure 4

Mean intensities in different resolution shells as a function of dose for the data from xtal01 recorded at $12.66 \mathrm{keV}$. The average intensity in the total range is displayed in black. All other curves show the decay for consecutive resolution intervals which are indicated to the left; for example, the resolution range is $30.0-3.10 \AA$ for the top curve (violet) and 3.10-2.45 $\AA$ for the second curve (blue). 
unit-cell parameters and $B$ factors as a damage metric, they found no clear dependence of damage on photon energy. Weiss et al. (2005) observed no significant difference in the radiation damage to crystals of a $\mathrm{Cd}$ derivative of elastase at wavelengths of 1.0 and $2.0 \AA$ (12.4 and $6.2 \mathrm{keV}$, respectively).

Our results indicate that the $D_{70}$ dose limit is about $11 \mathrm{MGy}$ at 12.66 and $19.0 \mathrm{keV}$, about three times smaller than the value of 30 MGy derived by Owen et al. (2006) at the same resolution. We do observe a different $D_{70}$ dose limit of $7.5 \mathrm{MGy}$ at $6.33 \mathrm{keV}$, which suggests that at low energy (long wavelength) thaumatin crystals are more susceptible to radiation damage. The average $D_{50}$ dose limits are 12.8, 20.4 and 20.0 MGy at $6.33,12.66$ and $19.0 \mathrm{keV}$, respectively.

In a recent study (Zeldin et al., 2013), $D_{50}$ values varied from 7 to $42 \mathrm{MGy}$ depending on how the dose was estimated. The authors conclude that the 'diffraction-weighted dose' is the most efficient metric, and report a value of $12.9 \mathrm{MGy}$ for insulin crystals at a resolution of $1.8 \AA$, which is also significantly lower than the value reported by Owen and coworkers.

As a side issue, we noted that the average intensity of reflections in the first image varied by up to a factor of two between the five crystals measured at each X-ray energy. Large variations in diffractive efficiency are often observed when different parts of a crystal are probed with a finely focused beam. Assuming that this is the result of a more perfect crystalline order and considering that one contribution to intensity decay is the corruption of the crystal lattice with increasing dose, it might be interesting to know whether higher diffractive efficiency results in higher dose tolerance. In Table 3, the relative diffractive efficiency derived by normalizing the average intensity of the first image by flux, exposure time and irradiated crystal volume is listed for each crystal. Note that comparison between the calculated values is only valid between crystals measured at the same energy. There seems to be no apparent correlation between diffractive efficiency and the $D_{70}$ or $D_{50}$ doses.

\section{Conclusions}

Recording a series of diffraction images at the same crystal orientation makes it possible to quantitatively assess the effect of X-ray radiation damage on the intensities of diffracted reflections, simultaneously minimizing the influence of various potential sources of error such as the sphere of confusion of the goniostat, imperfect centering of the crystal, residual nonuniformity of detector response or non-uniformly irradiated crystal volume when integrating over large rotation angles. This experiment, which was conducted on several crystals of thaumatin at three different $\mathrm{X}$-ray energies $(6.33,12.66$ and $19.0 \mathrm{keV})$, resulted in the estimation of the $D_{70}$ dose that reduces the average intensity of reflections to $70 \%$ of the initial value. The $D_{70}$ for reflections within a resolution range of 30-2.45 A was estimated to be about 11 MGy for 12.66 and $19.0 \mathrm{keV}$ radiation and $7.5 \mathrm{MGy}$ for $6.33 \mathrm{keV}$ radiation. These results suggest a $D_{70}$ dose limit that is about three times smaller than the value of $30 \mathrm{MGy}$ estimated by Owen $e t$ al. (2006) for a similar wavelength and resolution. This study suggests that there is no universally applicable dose limit for all types of protein crystals.

\section{Acknowledgements}

This project was supported in part by the Intramural Research Program of the National Cancer Institute, Center for Cancer Research and with Federal funds from the National Cancer Institute, National Institutes of Health (Contract No. HHSN261200800E). The content of this publication does not necessarily reflect the views or policies of the Department of Health and Human Services, nor does the mention of trade names, commercial products or organizations imply endorsement by the US Government. Diffraction data were collected on beamline 19-ID of the Structural Biology Center at the Advanced Photon Source, Argonne National Laboratory operated under contract DE-AC02-06CH11357 of the Department of Energy, Office of Biological and Environmental Research. Use of the Advanced Photon Source was supported by the US Department of Energy, Office of Science, Office of Basic Energy Sciences under Contract No. W-31-109Eng-38.

\section{References}

Alkire, R. W., Rosenbaum, G. \& Evans, G. (2000). J. Synchrotron Rad. 7, 61-68.

Blake, C. C. F. \& Phillips, D. C. (1962). Biological Effects of Ionizing Radiation at the Molecular Level, pp. 2-6. Vienna: International Atomic Energy Agency.

Burmeister, W. P. (2000). Acta Cryst. D56, 328-341.

Fourme, R., Honkimäki, V., Girard, E., Medjoubi, K., Dhaussy, A.-C. \& Kahn, R. (2012). J. Appl. Cryst. 45, 652-661.

Garman, E. F. (2010). Acta Cryst. D66, 339-351.

Garman, E. \& Nave, C. (2002). J. Synchrotron Rad. 9, 327-328.

Henderson, R. (1990). Proc. R. Soc. London Ser. B, 241, 6-8.

Hendrickson, W. A. (1976). J. Mol. Biol. 106, 889-893.

Holton, J. M. (2009). J. Synchrotron Rad. 16, 133-142.

Homer, C., Cooper, L. \& Gonzalez, A. (2011). J. Synchrotron Rad. 18, 338-345.

Hope, H. (1988). Acta Cryst. B44, 22-26.

Kmetko, J., Husseini, N. S., Naides, M., Kalinin, Y. \& Thorne, R. E. (2006). Acta Cryst. D62, 1030-1038.

Liebschner, D., Dauter, M., Rosenbaum, G. \& Dauter, Z. (2012). Acta Cryst. D68, 1430-1436.

McMaster, W. H., Kerr Del Grande, N., Mallett, J. H. \& Hubbel, J. H. (1969). Periodic Table: X-ray Properties. http://www.csrri.iit.edu/ periodic-table.html.

Otwinowski, Z. \& Minor, W. (1997). Methods Enzymol. 276, 307-326.

Owen, R. L., Rudiño-Piñera, E. \& Garman, E. F. (2006). Proc. Natl Acad. Sci. USA, 103, 4912-4917.

Paithankar, K. S., Owen, R. L. \& Garman, E. F. (2009). J. Synchrotron Rad. 16, 152-162.

Ravelli, R. B. G. \& Garman, E. F. (2006). Curr. Opin. Struct. Biol. 16, 624-629.

Ravelli, R. B. G. \& McSweeney, S. M. (2000). Structure, 8, 315-328.

Rodgers, D. W. (1994). Structure, 2, 1135-1140.

Rosenbaum, G. et al. (2006). J. Synchrotron Rad. 13, 30-45.

Shimizu, N., Hirata, K., Hasegawa, K., Ueno, G. \& Yamamoto, M. (2007). J. Synchrotron Rad. 14, 4-10.

Sliz, P., Harrison, S. C. \& Rosenbaum, G. (2003). Structure, 11, 13-19. Warkentin, M., Hopkins, J. B., Badeau, R., Mulichak, A. M., Keefe, L. J. \& Thorne, R. E. (2013). J. Synchrotron Rad. 20, 7-13. 


\section{research papers}

Warkentin, M. \& Thorne, R. (2010). J. Struct. Funct. Genomics, 11, 85-89.

Weik, M., Ravelli, R. B. G., Kryger, G., McSweeney, S., Raves, M. L., Harel, M., Gros, P., Silman, I., Kroon, J. \& Sussman, J. L. (2000). Proc. Natl Acad. Sci. USA, 97, 623-628.
Weiss, M. S., Panjikar, S., Mueller-Dieckmann, C. \& Tucker, P. A. (2005). J. Synchrotron Rad. 12, 304-309.

Zeldin, O. B., Brockhauser, S., Bremridge, J., Holton, J. M. \& Garman, E. F. (2013). Proc. Natl Acad. Sci. USA, 110, 2055120556. 